\title{
Persistence of conditioned fighting in a hen pigeon
}

FRANK N. WILLIS, JR., GERALDIHE MICHAEL AND JOSEPH EDWARDS

UNIVERSITY OF MISSOURI AT KANSAS CITY

Fighting a variety of opponents was observed in a hen pige on 11 months after conditioning to peck another bird. The hen fought and dominated three opponents but failed to fight a cock which displayed a pre-mating behavioral pattern. Frequency of fighting was found to be related to territoriality. Fighting patterns were frequently not typical of what has been described in the literature as stereotyped fighting in pigeons.

Conditioned fighting in pigeons has been studied by Reynolds et al (1963). They found that once fighting had been conditioned it continued in a stereotyped manner independent of the reinforcer. Fighting in pigeons has been related to available space, to age, and to prefight behavioral patterns by Willis (1966). All birds in these studies were cocks. Levi (1957) restricts his discussion of fighting in pigeons to cocks and in a recent statement said that hens do not fight (Levi, 1966).

The present study was designed to investigate the persistence of conditioned fighting in pigeons, to study fighting in hens, and to compare fighting in established territory with that in unfamiliar space. The study was initiated when a hen was observed to attack other birds 11 months after conditioned pecking of another bird was discontinued.

Method

Subjects. The primary subject (S) was a modena pigeon hen, $273 \mathrm{gm}$ in weight and 8-1/2 in. tall. Her opponents were a Carneaux cock (MC), a racing homer cock (MH), a modena cock (MM), and a modena hen (FM). Opponents ranged in weight from 376 to $\mathbf{5 3 5}$ gmand in height from $9 \mathrm{in}$. to $11-1 / 2$ in. All opponents were experimentally naive. All birds had 1964 or 1965 seamless bands.

Apparatus. Fighting was observed in three cages. Cages $A$ and $B$ were 28 in. wide, 34 in. long, and 29 in. high. Cage A was the home cage for S; the other cages were not used as housing. Cage $C$ was 16 in. wide, 16 in. long, and $9 \mathrm{in}$. high. Response times were recorded with a stop watch.

Procedure. S was conditioned to peck another pigeon (a racing homer cock) in an experimental chamber similar to that described by Ferster \& Skinner (1957). $S$ was maintained on $80 \%$ body weight during conditioning and was reinforced on an FR 1 schedule with grain. Pecking the other bird was reinforced for three 1-min. periods per day for 60 days. Training was discontinued and $S$ was not used as a subject for 11 months but food was available only $1 \mathrm{hr}$. each day. It was then observed that $S$ would immediately attack any bird that we attempted to house with her. It was decided to investi- gate this fighting with a variety of opponents and situations. S was paired in six sessions with four opponents in three cages for a total of 72 sessions. One session of $5 \mathrm{~min}$. duration was observed in each of the three cages in the morning and again in the afternoon. Placement in the cages was alternated so that $S$ or her opponent was first an equal number of times. Each cage was used first, second, and third an equal number of times in the morning and afternoon. Definitions of fighting responses and recording procedures were similar to those described by Willis (1966).

Results

S completely dominated three opponents. Her total number of fighting responses and those of her opponents were as follows: S-178, MH-0; S-131, MM-13; and S-140, FM-12. In cages $A$ and $B$ fighting occurred most frequently in early pairings and decreased in later pairings. Opponents after early sessions remained stationary in the cage while $S$ moved freely throughout the cage. Only when an opponent moved was fighting resumed. S's total fighting responses for the first through the sixth pairing in Cage A were $114,115,38,14,9$ and 8 . The six totals for Cage B were 35, 11, 19, 20, 1 and 0 . Territoriality in Cage $C$ was not established until the sixth pairing. The fighting response totals for this cage were $30,11,10,17,22$ and 0 . With opponent MC the response of $\mathrm{S}$ was very different. From the first session in which they were paired until the last, MC coaxed or strutted throughout the session. A detailed description of this pattern may be found in Levi (1957). $S$ responded to MC with a pre-mating pattern quite typical of hens. She walked around the cage with a strutting step, never attempting to escape and never attacking. MC pecked $S$ on one occasion.

Territoriality. S fought most frequently in her home cage (A) while opponents fought most in Cage C. Frequency of fighting responses for the three cages were as follows: for S, 290 in A, 82 in B, 77 in C; for opponents, 8 in $A, 4$ in $B, 13$ in $C$. A chi-square analysis yielded a $\chi^{2}=19.51$ with $d f=2$, and $p<.01$. A second datum related to territoriality was obtained from a comparison of frequencies of fighting responses when $S$ was placed in a cage first and when the opponent was placed in the cage first. $S$ tended to fight more frequently when she was first in the cage (269 to 180 fighting responses).

Fighting Response Pattern. S was conditioned only for pecking another bird. The terminal fighting which evolved, however, included more than this reinforced response. Similar results were reported by Reynolds et al (1963). They concluded that pigeons fight in a highly 
stereotyped pattern. Levi (1957) also describes fighting in pigeons as stereotyped. In the present study $\mathrm{S}$ developed an unusual style of fighting. She used wing slaps offensively and, in a manner somewhat like fighting cocks, she often pecked or slapped while in a mid-air rush at her opponent.

Finally, fighting for three sessions each morning and afternoon apparently did not tire S. Her total number of fighting responses for third sessions was almostidentical to her total for first sessions.

Discussion

The persistence of conditioned fighting was demonstrated by the appearance of a high rate of fighting responses 11 months after training. The fighting observed differed from conditioned fighting in that it included a more complex pattern than that conditioned. The unusual fighting responses that $S$ often displayed appears to require a further consideration of the degree of stereotype in the fighting of birds.

Contrary to the information in the literature, hen pigeons do indeed fight. Not only did S fight and dominate cock opponents but her hen opponent performed in a manner quite similar to that of the cocks. It is inter- esting to note that although conditioned fighting is persistent it can be completely eliminated by a premating display. In a former study (Willis, 1966) fighting between cocks was more likely to occur when coaxing first occurred. In this study the hen never fought when coaxing first occurred. There are sexdifferences in the fighting of pigeons but they are more complex than the present literature indicates.

This study provides additional information about territoriality in the fighting of birds. Familiarity with space and first occupancy appear more important than the size of available space as previously reported (Willis, 1966).

\section{References}

Ferster, C. B., \& Skinner, B. F. Schedules of reinforcement. New York: Apple-Century-Crofts, 1957.

Levi, W. The pigeon. Columbia, S. C.: Bryan, 1957.

Levi, W. Questions and answers. Amer. Pigeon J., 1966, 55, 41-42. Reynolds, G. S., Catania, A. C., \& Skinner, B. F. Conditioned and unconditioned aggression in pigeons. J. exp. Anal. Behav., 1963, 6, 73-74.

Willis, F. N. Fighting in pigeons relative to available space. Psychon. Sci., 1966, 4, 315-316. 